\title{
MIXED VEHICLE FLOW AT SIGNALIZED INTERSECTION: MARKOV CHAIN ANALYSIS
}

\author{
Ilya B. Gertsbakh \\ Department of Mathematics, Ben Gurion University, Israel \\ E-mail: elyager@bezeqint.net
}

\begin{abstract}
We assume that a Poisson flow of vehicles arrives at isolated signalized intersection, and each vehicle, independently of others, represents a random number $X$ of passenger car units (PCU's). We analyze numerically the stationary distribution of the queue process $\{Z n\}$, where $Z n$ is the number of PCU's in a queue at the beginning of the $n$-th red phase, $n \rightarrow \infty$. We approximate the number $Y_{n}$ of PCU's arriving during one red-green cycle by a two-parameter Negative Binomial Distribution (NBD). The well-known fact is that $\{Z n\}$ follow an infinite-state Markov chain. We approximate its stationary distribution using a finite-state Markov chain. We show numerically that there is a strong dependence of the mean queue length $E[Z n]$ in equilibrium on the input distribution of $Y_{n}$ and, in particular, on the "over dispersion" parameter $\gamma=\operatorname{Var}\left[Y_{n}\right] / E\left[Y_{n}\right]$. For Poisson input, $\gamma=1 . \gamma>1$ indicates presence of heavy-tailed input. In reality it means that a relatively large "portion" of PCU's, considerably exceeding the average, may arrive with high probability during one red-green cycle. Empirical formulas are presented for an accurate estimation of mean queue length as a function of load and $g$ of the input flow. Using the Markov chain technique, we analyze the mean "virtual" delay time for a car which always arrives at the beginning of the red phase.
\end{abstract}

Keywords: signalized traffic light, Markov chain, average queue length, mixed vehicle input flow, negative binomial distribution, over dispersed input, virtual delay

\section{Introduction. Model Description}

We consider one-lane vehicle flow arriving at a signalized intersection working in periodic regime. One cycle consists of a green phase of duration $T_{g}$ followed by a red phase of duration $T_{r}$. We will use index $n$ to denote the $n$-th working cycle, $n=1,2, \ldots$ Let $X_{n}$ be the number of vehicles arriving at the intersection during the $n$-th cycle.

It will be assumed that $\left\{X_{n}\right\},(n=1,2, \ldots$,$) is a sequence of independent identically distributed$ random variables (r.v.'s) following the Poisson distribution with parameter $\Lambda$. Contrary to the standard assumption about the input flow, we assume that vehicles differ in their geometric parameters, so that from the traffic load point of view they represent different numbers of PCU's (passenger car units). For example, the text (Slinn et al., 2005, 102) gives the following data:

$\begin{array}{ll}\text { Vehicle type } & \text { PCU } \\ \text { Cars and light goods vehicles } & 1 \\ \text { Medium goods vehicles } & 1.5 \\ \text { Buses and coaches } & 2.0 \\ \text { Heavy goods vehicles } & 2.3\end{array}$

More formally, it will be assumed that the $i$-th vehicle, independently of other vehicles in the input flow, represents $V_{i}$ PCU's, and

$P\left(V_{i}=w_{j}\right)=p_{j}, j=1, \ldots, k$.

If, for example, $80 \%$ of all vehicles are passenger cars, $10 \%$ - buses, and the rest - heavy goods vehicles, then $w_{1}=1, w_{2}=2, w_{3}=2.3$, and $p_{1}=0.8, p_{2}=0.1, p_{3}=0.1$.

Now the number of PCU's arriving at the intersection during $n$-th traffic light cycle is represented as

$Y_{n}=V_{1}+V_{2}+\ldots+V_{X n}$,

i.e. as a sum of random number of random variables. It is assumed that the r.v.'s $X_{n}$ and $V_{i}$ are independent. 
The standard Poisson input flow of cars is a particular case of (2) if $P\left(V_{i}=1\right)=1$, i.e. if each vehicle represents one PCU.

Random variable $Y$ (we will omit the cycle index $j$ ) has a compound Poisson distribution, see Feller (1963), Chapter 12. Its mean value equals

$E[Y]=E\left[V_{i}\right] \cdot E[X]=E\left[V_{i}\right] \cdot \Lambda$

It is assumed that during the green phase, the intersection can serve the maximal number $m$ of PCU's and that the intersection works in stationary (underflow) regime for which the load

$\rho=\frac{E[Y]}{m}<1$

Our main goal is to analyze the number of PCU's $Z_{n}$ waiting in line at the beginning of red phase in the $n$-th cycle. The case that all vehicles in the input flow represent one PCU (one car) was investigated by Newell (1960) in his classic work. He postulated the following principal relationship between $Z_{n+1}$ and $Z_{n}$ :

$Z_{n+1}=\max \left[Z_{n}+Y_{n+1}-m, 0\right]$

This formula means the following: if the number of cars left unserved from the previous cycle $Z_{n}$ plus the number of cars $Y_{n+1}$ arrived at the intersection during the present cycle (no matter what the exact arrival times were) is $\geq m$, the number of cars passed through the intersection will be $m$. Thus the number of cars in queue at the beginning next red phase will be $Z_{n+1}$ according to (5). The importance of (5) lies in the fact that it establishes that the sequence of r.v.'s $\left\{Z_{n}\right\},\{n=1,2,3, \ldots\}$ creates a Markov chain. This fact is crucial to the theoretical and numerical investigation of the queue length in equilibrium.

Two complications arise when we are dealing with the input flow of vehicles having different number of PCU's. The first one is that now $Y_{n}$ are not always integer-valued. If, for example, 8 "regular" cars, one bus and one light good truck arrive during one cycle, the number of PCU's will be 10.5.

The second one is that the maximal number of $m$ PCU 's not always can be implemented. Imagine, that in the above example the bus (2 PCU's) is the last vehicle approaching the intersection during the green phase. Depending on its driver's habit, the bus may start moving across the intersection even if in the middle of its movement the light will change to red. In this way the intersection provides an extra service capacity of about one PCU. On the contrary, the more hesitant driver would not start moving across the intersection, leaving one time interval of length $\delta=T g / m$ unused. We decided to ignore the second complication by assuming that in the long run, the "under use" and "over use" of maximal capacity $m$ compensate each other.

As to the first complication, we assume that the actual number of PCU's in one cycle can be rounded to the nearest integer. So, for example, if $X_{n} \in[9.5,10.5)$, we put $X_{n}=10$. If the round-off error is uniformly distributed in the interval $[-0.5,0.5]$, the average error $e$ of the round-off by its absolute value is equal 0.25 ; for a triangular distribution of the error $e=0.33$. In our opinion, it can be neglected if the average number of PCU's per cycle is about 8-12. Besides, only a part of all cycles have non integer values of PCU's.

In this paper, our task is calculating the mean number of PCU's $Q$ waiting in the queue at the beginning of red light. As a rule, the calculation of the mean delay at the traffic light demands the knowledge of $Q$, see, e.g. the works of Newell (1960, 1965), McNeill (1968), Darroch (1963), Miller (1963), and Webster (1958). As a rule, these works take into account also the variability of the input flow by introducing the "over-dispersion" parameter $\gamma=\operatorname{Var}[Y] / E[Y]$.

The further exposition will be as follows. In Section 2 we investigate the variance of r.v.'s $Y_{n}$ and discuss the possibility to approximate their distribution by the Negative Binomial (NBD). Its important property is that it allows to describe the input flow for the so-called over dispersed case of $\gamma>1$. NBD is a "heavy-tailed" distribution comparing to Poisson. In Section 3 we investigate numerically the stationary distribution of $Z_{n}$ by means of Markov chain technique and present the queue parameters for various loads and the Poisson input flow of vehicles. In Section 4 we present numerical results for a collection of input flows, for various loads $\rho$ and various degrees of "over dispersion" $\gamma$ and compare the queue parameters with the Poisson input flow. We demonstrate that "over dispersed" input flow considerably increases the queue length and, therefore, the delay time. In Section 5 we introduce the notion of virtual delay time and present formulas and numerical results for its mean and variance for various input flows. The appendix 
contains a collection of empirical formulas expressing the mean queue length as a function of $\rho$ and $\gamma$ and compares them to Miller's approximation (1963).

\section{Variance of $Y_{n}$. Negative Binomial Distri- bution (NBD)}

By the result of (Feller, 1963), Chapter 12, Section 6,

$\operatorname{Var}[Y]=E[X] \cdot \operatorname{Var}[V]+\operatorname{Var}[X] \cdot E^{2}[V]=\Lambda \cdot E\left[V^{2}\right]$.

Comparing with (3), we see that

$\operatorname{Var}[Y] / E[Y]=E\left[V^{2}\right] / E[V]>1$,

while for "regular" Poisson input flow this ratio equals 1 . It has been noticed already in the early traffic investigations (Darroch, 1963; Miller, 1963; Webster, 1958) that over dispersion considerably increases the car delay time at the intersection.

In the above example of mixed flow with $10 \%$ of buses and $10 \%$ of heavy goods vehicles,

$E\left[V^{2}\right]=1 \cdot 0.8+2^{2} \cdot 0.1+2.3^{2} \cdot 0.1 \approx 1.53$

which is a rather large increase in variability of the number of PCU's per cycle compared to the Poisson flow.

To carry out the further investigation of the Markov process $\left\{Z_{n}\right\}$ we must have an analytic form of distribution of $Y_{n}$. In statistical practice, the so-called Negative Binomial distribution (NBD) is considered a suitable candidate for an over dispersed distribution. Its probability mass function has the following form:

$g(k)=[\operatorname{Gamma}(k+r) / \operatorname{Gamma}(r) k !](1-p)^{k} p^{r}$

for $k=0,1,2, \ldots$

Table 1: Fitting NBD to Mixed Input Flow. $\Lambda=10$

\begin{tabular}{lllllllll}
\hline No/PCU & $w_{1}=1$ & $w_{2}=2$ & $w_{3}=3$ & $w_{4}=4$ & $r$ & $p$ & $\gamma$ & $\mathrm{X}^{2}$ \\
\hline 1 & 0.7 & 0.25 & 0.05 & & 16.2 & 0.54 & 1.8 & 25.3 \\
2 & 0.8 & 0.15 & 0.05 & & 17.6 & 0.58 & 1.7 & 25.9 \\
3 & 0.9 & 0.075 & 0.025 & & 27.5 & 0.71 & 1.4 & 12.2 \\
4 & 0.95 & 0.025 & 0.025 & & 36.2 & 0.77 & 1.3 & 20.6 \\
5 & 0.95 & 0.025 & 0.02 & 0.005 & 31.2 & 0.74 & 1.3 & 26.7 \\
$\mathbf{6}$ & $\mathbf{0 . 9}$ & 0 & 0 & $\mathbf{0 . 1}$ & 8.41 & 0.39 & $\mathbf{2 . 5 4}$ & $\mathbf{3 9 . 7}$ \\
7 & 0.9 & & 0.1 & & 15.0 & 0.56 & 1.8 & 28.5 \\
8 & 0.9 & 0.1 & & & 41.7 & 0.79 & 1.3 & 21.2 \\
\hline
\end{tabular}

Here $r$ is a real nonnegative parameter, and $p \in(0,1)$. For $r \rightarrow \infty$, NBD approaches Poisson distribution. follows:

Two central moments of the NBD, mean $\mu$ and variance $V a r$, are expressed via parameters $p, r$ as

$\mu=r(1-p) / p, \quad \operatorname{Var}=\mu(1+\mu / r)$.

There is only one situation where the Poisson sum of integer-valued r.v.'s exactly follow the NBD. This is the case when the random summands have so-called discrete logarithmic distribution, with probability mass function $f_{n}=C \cdot a^{n} / n, n=1,2, \ldots$, see (Feller, 1963), Chapter 12. Unfortunately, this distribution does not describe well the distribution of PCU's in a single vehicle.

Our further analysis of the mixed input flow is based on the following assumption: the total number of PCU's in the vehicles arriving during one cycle (after round-off as described above) can be approximated by an NBD having the same two central moments, i.e. the same mean and variance. In other words, we 
assume that the distribution of $Y_{n}$ approximately follows the NBD. We remind that $Y_{n}$ and $Z_{n}$ represent now not the number of vehicles, but the number of PCU's arriving during one cycle at the traffic light and the number of PCU's waiting in queue at the beginning of red light, respectively.

Table 1 presents a series of eight simulation results, each based on 500 independent replicas of simulated r.v.'s $Y_{n}$. Each replica represents an observed value of the total number of PCU's in $X$ arriving vehicles, where $X$ is a Poisson r.v. with mean $\Lambda=10$.

Columns 2-5 present the probabilities of having $w_{1}, \ldots, w_{4}$ PCU's in one vehicle. For example, in case No 3, 90\% of all vehicles are passenger cars (1 PCU), 7.5\% have - two and 2.5\% - three PCU's. $r=27.5$ and $p=0.71$ are the parameters of the NBD obtained by fitting the mean and variance of $Y_{n}$ to the mean and variance of the NBD. In this example, the "over dispersion" parameter $\gamma=\operatorname{Var}[Y] / E[Y]$ is 1.4.

The last column is the observed value of the chi-square statistic. In our experiment, we worked with 30 frequencies united into 22 groups, which corresponds to 21 degrees of freedom. The critical values are $\chi_{0.2}^{2}=26.2, \quad \chi_{0.1}^{2}=29.6, \chi_{0.05}^{2}=32.7$. We see, therefore, that in all cases except the case No 6, we observe an excellent fit. The tendency is that the quality of the fit decreases when the number of PCU's becomes more dispersed. Physically, it happens with the increase of the percentage of very long vehicles. So, in case 6 we have a bad fit because of a relatively large percentage of very long vehicles with $w_{4}=4$, which results in $\gamma=2.54$, much higher than in other cases.

\section{Stationary Distribution of $\{Z n\}$}

The Markov chain of eqn (5) has an infinite one-step transition matrix $\mathbf{P}=\left\|p_{i, j}\right\|, i, j=0,1,2, \ldots$, , where $p_{i, j}=P\left(Z_{n+1}=j \mid Z_{n}=i\right)$. We remind that $Z_{n}$ is the number of PCU's in the line at the beginning of the $n$-th red light phase.

Let us determine the elements of $\mathbf{P}$. Denote by $G(k)=P(Y \leq k), g(k)=P(Y=k), k \geq 0, g(k)=0$ for $k<0$. (Here index $n$ is omitted for the r.v.'s $Y_{n}$.) The elements of matrix $\mathbf{P}$ depend on $m$ and on the discrete density $g(k)$ :

$p_{i, 0}=G(m-i), i=0,2 ., \ldots, m ; p(i, 0)=0$, if $i>m$

$p_{i, j}=g(m+j-i)$, for $i=0,1,2, \ldots, j>0$.

For example, suppose that $m=5, i=6, j=1$, i.e. $Z_{n}=6, Z_{n+1}=1$. This happens if and only if no new cars arrive during the cycle, i.e. with probability $p_{6,1}=g(0)$. Let us determine $p_{86}=P\left(Z_{n+1}=6 \mid Z_{n}=8\right)$. Since $8+Y-m=6$ implies that $Y=3, p_{86}=g(3)$.

Interestingly, that a transition matrix identical to $\mathbf{P}$ has been introduced in [4], Section 5.9, for car delay in the model of group service, see also (Gertsbakh, 2008; Newell, 1960). Denote by $D=\left\{\pi_{0}, \pi_{1}, \pi_{2}, \ldots,\right\}$ the stationary distribution for the Markov chain with matrix P. Consider a modified truncated Markov chain which has $M$ states $0,1, \ldots, M-1$ by placing a semi-reflecting barrier in the state $M$. Formally, this means considering an $(M)$-state chain with the one step transition matrix $\mathbf{P}_{\mathbf{M}}=\left\|p_{i, j}(M)\right\|$, where $p_{i, j}(M)=p_{i, j}$ for $i=0,1, \ldots, M-1, j=0,1, \ldots, M-2$ and $p_{i, M-1}=1-\sum_{j=0}^{M-2} p_{i, j}$ for $0=1,2, \ldots, M-1$. In practice, we take $M>>m$, and the probabilities $p_{i, j}$ are very small for $j \geq M$. Physically, the truncated matrix $\mathbf{P}_{\mathbf{M}}$ describes a random walk which behaves for the states $i=0,1,2, \ldots, M-2$ exactly as the original Markov chain. When it is in state $i$, it goes to $M-1$ with probability $p_{i, M-1}$, or jumps "backward" into lower state $k, k<i$ with probability $p_{M-1, i}$. This truncated chain describes operation of an intersection with an extra agreement: when the queue reaches (high) level $M-1$, in the next cycle the intersection will not be entered by the number of cars exceeding $m$.

Table 2: Queue parameters for Poisson input $Y$

\begin{tabular}{l|l|l|l|l|l|l|ll}
\hline Parameter & $\rho=$ & 0.7 & 0.75 & 0.8 & 0.85 & 0.9 & 0.925 & 0.95 \\
\hline Average Qavr & & 0.25 & 0.45 & 0.80 & 1.47 & 2.98 & 4.56 & 7.76 \\
St. dev. $\sigma Q$ & & 0.90 & 1.27 & 1.84 & 2.80 & 4.53 & 6.3 & 9.5 \\
$\pi_{0}=P(Z n=0)$ & & 0.894 & 0.833 & 0.747 & 0.629 & 0.472 & 0.375 & 0.265 \\
\hline
\end{tabular}

Denote by $D_{M}=\left\{\pi_{0}(M), \pi_{1}(M), \ldots, \pi_{M}(M)\right\}$ the equilibrium distribution for the truncated chain. We state without proof the following 
Claim. Denote by $\Delta$ the Euclidian distance between $D_{M}$ and $D$.

Then $\Delta$ tends to zero as $M$ tends to infinity. \#

The choice of $M$ for the truncated Markov chain is dictated by the geometry of the link upstream of the intersection, see e.g. Viti (2006). We assume that each PCU occupies 6 meters on the lane along which it moves. Leaving only 1 meter free space between the vehicles in the queue, and assuming that the next upstream intersection is about 500 meters away, we arrive at the value of $\mathrm{M}=70$.

\section{Remark.}

It is convenient to calculate the stationary distribution $D(M)$ by raising the matrix $\mathbf{P}(M)$ to power $k=200-400$. By the properties of Markov chains, all rows of the matrix $\mathbf{W}=[\mathrm{P}(\mathrm{M})]^{\mathrm{k}}$ represent the equilibrium distribution.\#

We conclude this section by numerical results for Poisson input of PCU's (each car $=1 \mathrm{PCU}$ ), for various load values $\rho$. We consider typical intersection with $T_{g}=T_{r}=36 \mathrm{sec}, m=12$ and $\delta=36 / 12=3 \mathrm{sec}$ interval to service one car. We have carried out the numerical investigation for the truncated matrix $\mathbf{P}(M)$, assuming $P(Y=k)=e^{-\Lambda} \cdot \Lambda^{k} / k !, k=0,1,2, \ldots$ The load $\rho=\Lambda /(m=12)$. The results are presented in Table 2 for $\rho=0.70(0.05) 0.90,0.925,0.95$.

\section{Numerical Investigation of the Queue for NBD Input Flow}

In this section we consider a typical signalized intersection with the red phase of duration $T_{r}=36 \mathrm{sec}$ and green phase of $T_{g}=36 \mathrm{sec}$. The maximal number $m$ of cars served during the green phase is assumed to be $m=12$. The distribution of the number of PCU's per cycle is assumed to be NBD.

We consider several examples of load values $\rho=E[Y] / m \in[0.7,0.95]$ and degrees of over dispersion $\gamma=\operatorname{Var}[Y] / E[Y] \in[1.25,2.5]$.

The main parameter of our interest is the average queue length $Q_{N B D}$. The central qualitative result of this paper is the fact that $Q_{N B D}$ considerably exceeds the average queue length $Q_{a v r}$ for the Poisson input flow.

It will be extremely convenient to express the parameters of NBD, $p$ and $r$, via the load $\rho$ and $\gamma$. It is easy to obtain the following formulas:

$p=1 / \gamma, r=\rho m /(\gamma-1)$.

Since $m$ was assumed constant $(m=12)$, we considered the dependence of the average queue length $Q_{N B D}$ as a function of load $\rho$ for several fixed $\gamma$ values. If $\gamma$ is constant, $p$ is constant too, and the only parameter depending on $\rho$ is $r$. For each fixed pair $p, r$ we considered the corresponding Markov chain with the above described matrix $\mathbf{P}(M)$ and computed the average queue length $Q_{N B D}$ using the stationary distribution $\pi=\left\{\pi_{0}, \pi_{1}, \ldots, \pi_{M-1}\right\}$ :

$Q_{N B D}=E[Z]$.

We used in our calculations the following values of $\rho: 0.70,0.80,0.85,0.90,0.925$ and 0.95 . It turned out that the dependence of the average queue on $\rho$ is very well approximated by a function of type $(a+b \rho) /(1-c \rho)$. For example, for Poisson input flow

$Q_{a v r}=\frac{-0.7621+1.1596 \cdot \rho}{1-1.0068 \cdot \rho}$.

We obtained also approximations for fixed $\gamma=1.25,1.5,2,2.5$. The corresponding formulas are presented in the Appendix.

Our main results are presented in Fig. 1 in a form of a family of curves showing the queue length dependence on the load, for various $\gamma$ values.

Fig. 1 leads to the following conclusions.

(1) For a fixed value of $\gamma$, the queue length rapidly increases when load approaches to 1 .

(2) For the same load $\rho$, the average queue length for NBD is always considerably larger than the average queue length for Poisson input.

(3) For fixed $\rho$, the value of $Q_{N B D} / Q_{a v r}$ increases with the increase of $\gamma=\operatorname{Var}[Y] / E[Y]$. 


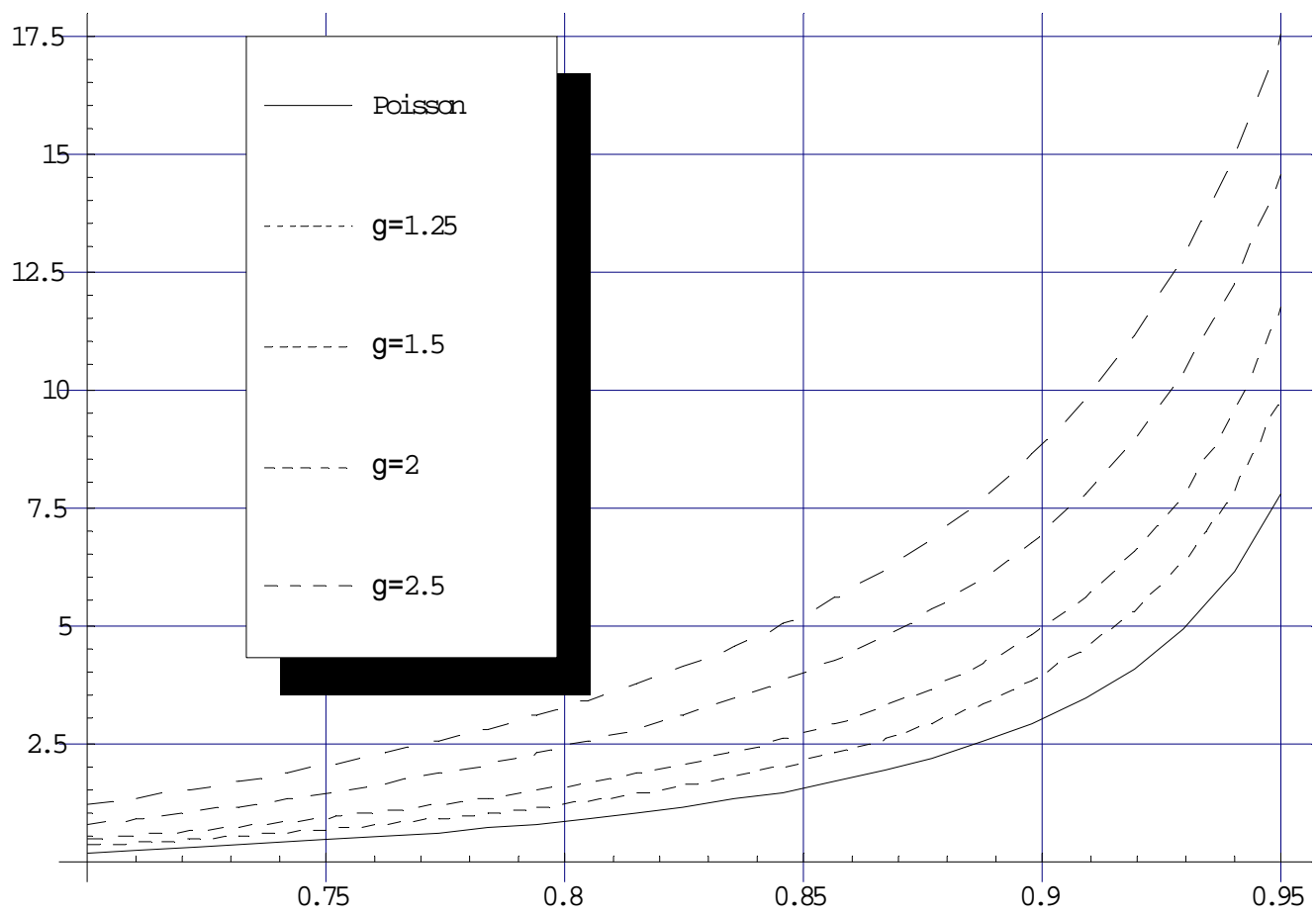

Figure 1. Average queue as function of load

Speaking in terms of vehicles in the input flow, the parameter $\gamma$ increases with the increase of the percentage of heavy/long vehicles (trucks, buses, trailers etc).

\section{Virtual Delay}

We define the "virtual delay" as the waiting time of a fictitious "yellow" car arriving at the intersection exactly at the instant when the green light changes to red. This car saw in front of him all $Z_{n}$ vehicles already waiting in line.

Deriving a formula for average delay and the variance of the delay of a car arriving at a random point in time is a rather involved issue and typically is made by assuming a specific model of car arrival process during the green-red phase, see e.g., (Darroch, 1963; Miller, 1963) and the discussion in (Viti, 2006). Contrary to this situation, it is very easy to calculate the mean and variance of the "yellow" delay time. The key is the fact that we know the stationary distribution of the queue $\left\{Z_{n}\right\}$.

If $Z_{n}=k$, then the yellow car waits $\theta_{k}=T_{r}+\delta+\operatorname{Integer}[\mathrm{k} / \mathrm{m}]\left(T_{r}+T_{g}\right)+(k-$ Integer $[\mathrm{k} / \mathrm{m}] \cdot \mathrm{m}) \cdot \delta$, where $\delta=T g / m$ is the service time of one PCU. Therefore, the mean virtual delay $\mu_{v}$ equals

$\mu_{v}=\sum_{k=0}^{M-1} \pi_{k} \cdot \theta_{k}$

Similarly, it is easy to find out the standard deviation $\sigma_{v}$ of the virtual delay:

$\left.\sigma_{v}=\left[\sum_{k=0}^{M-1} \pi_{k} \cdot \theta_{k}^{2}-\mu_{v^{2}}\right)\right]^{0.5}$.

The values of $\mu_{v}$ and $\sigma_{v}$ are presented in Table 3, for Poisson input and for NBD distribution of PCU's, for various values of parameter $\gamma=\operatorname{Var}[Y] / E[Y]$ and various loads $\rho$.

These results can be summarized as follows. Both $\mu_{v}$ and $\sigma_{v}$ increase with the increase of the load $\rho$. For fixed $\rho$, they increase significantly with the degree of over dispersion $\gamma$.

In our opinion, both virtual mean and standard deviation may serve as conservative ("worst case") characteristics of the delay at signalized intersection. We believe that the mean virtual delay exceeds the 
actual delay by at least $T_{r} / 2$ (about half of the vehicles arrive at green light and don't wait at all or wait very little) and about half of the vehicles wait time $T_{r}$.)

Another advantage of using the virtual delay is that it is easily adjusted to rather a realistic situation of variable input flow, i.e. to the case of non stationary input $Y_{n}$. To carry out this adjustment, it is necessary to take into account the varying distribution of $Y_{n+1}, n=s, s+1, \ldots$, and the present distribution of the queue $Z_{s}$. The Markov chain technique is applicable to this case if one takes into account that now the one-step transition matrix $P^{(s)}(M)$ will depend on the cycle numbers $s$. The analysis of non stationary input flow lies outside the scope of this paper.

Table 3: Mean Virtual Delay and Its Standard Deviation

\begin{tabular}{|c|c|c|c|c|c|c|}
\hline \multirow[t]{2}{*}{ Input flow } & \multicolumn{2}{|c|}{$\rho=0.7$} & \multicolumn{2}{|c|}{$\rho=0.85$} & \multicolumn{2}{|c|}{$\rho=0.95$} \\
\hline & $\mu \nu$ & $\sigma v$ & $\mu v$ & $\sigma v$ & $\mu v$ & $\sigma v$ \\
\hline Poisson & 39.7 & 2.7 & 43.9 & 11.2 & 74.8 & 53.0 \\
\hline NBD input, $\gamma=1.5$ & 40.5 & 4.9 & 48.9 & 27.3 & 97.5 & 76.5 \\
\hline NBD input, $\gamma=2.0$ & 42.2 & 10.1 & 54.9 & 31.6 & 116.3 & 92.0 \\
\hline NBD input, $\gamma=2.5$ & 44.0 & 14.7 & 61.4 & 41.7 & 130.8 & 101.9 \\
\hline
\end{tabular}

\section{Appendix}

Below are the formulas for the average queue length $Q_{N B D}$ for NBD input flow of PCU's:

For $\gamma=1.25, \quad Q(N B D)=(-0.9106+1.4341 \rho) /(1-1.0043 \rho)$,

For $\gamma=1.5, \quad Q(N B D)=(-1.0838+1.7487 \rho) /(1-1.0008 \rho)$,

For $\gamma=2.0, \quad Q(N B D)=(-1.6547+2.7043 \rho) /(1-0.0866 \rho)$,

For $\gamma=2.5, \quad Q(N B D)=(-1.9851+3.3623 \rho) /(1-0.9800 \rho)$.

These formulas estimate the average queue with an absolute error not exceeding 0.05-0.2.

In conclusion, let us note that formulas (16)-(19) are more accurate than rather popular Miller's approximation (1963).

\section{References}

1. Darroch, J.N. (1963) On the Traffic-Light Queue, Ann. Math. Statist., 15, 380-388.

2. Feller, W. (1963) An Introduction to Probability Theory and Its Applications, Vol.1, 2nd edition, John Wiley\& Sons, Inc.

3. Gertsbakh, I. (2008) Equilibrium waiting time and queue length on an intersection: group arrival of cars. Proceedings of International. Conference "Modeling of Business, Industrial and Transport Systems”, May 7-10, Riga, Latvia.

4. Haight, Frank, A. (1963) Mathematical Theories of Traffic Flow, Academic Press New York London.

5. McNeil, D.R. (1968) A solution to the fixed cycle traffic light with compound Poisson arrivals. Journal of Applied Probability, 5(3),624-635.

6. Miller, A.J. (1963) Settings for Fixed-Cycle Traffic Signals. Operational Research Quarterly, 14, 373-386. 1963.

7. Newell, G.F. (1960) Queues for a fixed-cycle traffic light, Annals of Mathematical Statistics, 31, 589-597.

8. Newell, G. (1965) Approximation Methods for Queues with Application to to Fixed Traffic Light. SIAM Review, 7.

9. Slinn, M., Guest, P, and P. Matthews. (2005) Traffic Engineering Design, Principles and Practice, 2nd edition, Elsevier.

10. Viti, F. T. (2006) The Dynamics and the Uncertainty of Delays at Signals. Ph.D. thesis, Delft University of Technology, TRAIL Press, The Netherlands.

11. Webster, F.V. (1958) Traffic signal settings. Paper No 39, Her Majesty Stationery Office, London. 